\title{
DE LA TUTORÍA A LA MENTORÍA. REFLEXIONES \\ EN TORNO A LA DIVERSIDAD DEL TRABAJO DOCENTE
}

From Tutoring to Mentoring.

Reflections on the Diversity of Teaching Functions

\author{
SALVADOR PONCE CEBALLOS*1 \\ BENILDE GARCÍA-CABRERO**2 \\ DENNISE ISLAS CERVANTES*3 \\ YESSICA MARTÍNEZ SOTO*4 \\ ARMANDINA SERNA RODRÍGUEZ*5 \\ *Universidad Autónoma de Baja California. México. \\ Correspondencia: ponce@uabc.edu.mx,dislas@uabc.edu.mx, \\ yessicamas@uabc.edu.mx, armandinaserna@uabc.edu.mx \\ **Universidad Nacional Autónoma de México. México. \\ Correspondencia: benildegar@gmail.com
}

Recibido: 14-12-2016

Revisado: 04-05-2017

Aceptado: 26-09-2017

Resumen: Las funciones docentes en las instituciones de educación superior se han diversificado para mejorar la terminalidad y prevenir el rezago académico. Si bien la tutoría ha sido hasta el momento la estrategia por excelencia, existen otras funciones docentes como la supervisión, la asesoría, la orientación educativa, el coaching educativo y, en especial, la mentoría, que ha sido poco empleada en el contexto educativo local. El propósito de este artículo es conceptualizar de manera teórica estas funciones docentes para diferenciarlas y mostrar cómo pueden complementarse. Se argumenta sobre el valor de la mentoría en la formación de los estudiantes y se concluye que es necesario incorporarla en las instituciones de educación superior, debido a su potencial para promover el logro académico.

Palabras clave: tutoría universitaria; funciones docentes; mentoría; orientación educativa; coaching educativo.

\footnotetext{
${ }^{1}$ Doctor en Educación. Profesor-Investigador de la Facultad de Pedagogía e Innovación Educativa en la Universidad Autónoma de Baja California (México). Línea de trabajo: formación del profesorado.

2 Doctora en Psicología. Profesora de la Facultad de Psicología en la Universidad Nacional Autónoma de México. Líneas de trabajo: evaluación y formación docente, diseño y evaluación curricular, el uso de las tecnologías digitales en la educación y el desarrollo moral y la educación cívica y ética.

${ }^{3}$ Doctora en Ciencias de la Educación. Académica de la Facultad de Pedagogía e Innovación Educativa en la Universidad Autónoma de Baja California (México). Líneas de trabajo: orientación educativa, tutorías y calidad de vida.

4 Doctora en Educación. Académica de la Facultad de Ciencias Humanas en la Universidad Autónoma de Baja California (México). Líneas de trabajo: la orientación educativa, tutoría, inteligencia emocional y violencia de género.

${ }^{5}$ Doctora en Educación. Académica de la Facultad de Pedagogía e Innovación Educativa en la Universidad Autónoma de Baja California (México). Líneas de trabajo: ética y valores profesionales, tutoría y formación del profesorado.
} 
Abstract: Teaching functions in higher education institutions have been diversified to improve education completion and prevent academic underachievement. While tutoring has so far been the main strategy, there are other teaching functions such as supervision, counselling, educational guidance, educational coaching and particularly mentoring, which at present have been little used in higher education contexts. The purpose of this article is to theoretically conceptualize these teaching functions to differentiate them and show how they can complement each other. This paper argues about the value of mentoring in the training of students, and concludes that it is necessary to incorporate it into higher education institutions because of its potential to promote academic achievement.

Keywords: college tutoring; teaching functions; mentoring; counselling; educational coaching.

\section{INTRODUCCIÓN}

Dentro de las funciones docentes que se han incorporado con más fuerza en el ámbito universitario destaca la tutoría. La actividad tutorial ha adoptado diversas formas y significados, focalizando su atención principalmente en dar respuesta a las necesidades emergentes de los estudiantes en las Instituciones de Educación Superior (IES). La tutoría ha sido conceptualizada como una actividad de corte psicopedagógico y por tanto de acompañamiento, guía y soporte para el estudiante.

En la Declaración Mundial sobre la Educación Superior en el siglo XXI (1998) propuesta por la UNESCO, se estableció la necesidad de modificar el proceso de aprendizaje en la Educación Superior, de adoptar una nueva visión y de desarrollar un nuevo modelo de enseñanza superior centrado en el alumno, asimismo se puntualizó que una de las responsabilidades del profesor es proporcionar orientación y consejo, cursos de recuperación, formación para el estudio y otras formas de apoyo requeridas por los alumnos con el fin de mejorar sus condiciones de vida.

La Asociación Nacional de Instituciones de Educación Superior (ANUIES) formuló en el año 2000 líneas estratégicas de desarrollo en las que plasmó su visión sobre el Sistema de Educación Superior (SES), así como las rutas por las que debía transitar en el mediano y largo plazos. A partir de dichas estrategias se generaron 14 programas, uno de ellos fue el de Desarrollo Integral de los Alumnos, que se propuso como objetivo apoyar a los alumnos con programas de tutorías y desarrollo integral, diseñados e implementados por las IES, con el fin de que una elevada proporción de ellos culminara sus estudios en el plazo previsto y lograra los objetivos de formación establecidos en los planes y programas de estudio (Romo, 2004).

En el año 2002, la ANUIES se propuso apoyar a las IES para que respondieran a los compromisos establecidos en el Programa de Mejoramiento al Profesorado (PROMEP), para ello convocó a un grupo de especialistas en el ámbito de la educación superior de diferentes instituciones con el fin de construir una propuesta para la 
organización e implantación de programas de atención personalizada para alumnos universitarios; generándose como resultado de este trabajo, una propuesta acorde con las líneas estratégicas de desarrollo para la educación superior.

Con el propósito de apoyar esta iniciativa, la ANUIES se propuso la ampliación y fortalecimiento de su programa de educación continua para ofrecer servicios de asesoría y capacitación a los responsables institucionales de diseñar y operar este tipo de programas, estableciendo mecanismos para su actualización y obteniendo así mayor eficiencia en los procesos de titulación, elevar la calidad del aprendizaje y mejorar los sistemas de evaluación para verificar el logro de esos objetivos.

La implementación del sistema tutorial en diversas IES mexicanas tiene variantes emergentes a partir de la necesidad de adecuar la tutoría a las condiciones institucionales considerando su historia, tradiciones, planta docente, modelo curricular, recursos humanos, el clima organizacional y sus dinámicas, ente otras; es por ello que la tutoría constituye una de las estrategias fundamentales correspondiente con la nueva visión de la educación superior, debido a que permite potenciar la formación integral del alumno con una visión humanista y responsable. Por tanto, resulta un recurso que facilita la adaptación del estudiante al ambiente escolar, mejora sus habilidades de estudio y hábitos de trabajo, contribuye a abatir los índices de reprobación y rezago escolar, disminuye las tasas de abandono de los estudios y mejora la eficiencia terminal, al atender puntualmente los problemas específicos de las trayectorias escolares en cada una de las dependencias académicas (Lara, 2009).

El tutor pone al servicio del alumno todas sus capacidades y cualidades para proveerle de información y orientarlo de forma objetiva y desinteresada al logro de sus proyectos académicos, impulsando su potencial, mostrándole opciones para la toma de decisiones y promoviendo su autonomía, apoyando así el propósito de formación integral propuesto en las IES (Islas y González, 2014).

\section{La problemática inherente a la definición e instrumentación de los procesos de tutoría}

Una de las misiones primordiales de las IES es la formación de individuos altamente calificados para responder, mediante su participación activa, a las demandas y necesidades individuales y sociales. A lo largo de su historia las universidades han desarrollado diversas estrategias que ya sea de manera alterna o paralela han venido a fortalecer el currículum coadyuvando al logro de su importante misión: la entrega a la sociedad de ciudadanos competentes y sensibles con el entorno, con un alto grado de competencia en la aplicación del conocimiento científico, pensamiento crítico, creativo e innovador que favorezca el desarrollo y mejora de las comunidades y del país. 
Una de las estrategias fundamentales para lograr esta misión sin duda alguna lo constituye la actividad tutorial, entendida como el proceso de acompañamiento estudiantil que implica una relación cercana entre el docente y el alumno, convirtiéndose en lo que Zabalza (2007) señala como una parte sustancial del perfil profesional del docente universitario. Por su parte, Canales (2010) menciona que la tutoría puede entenderse como una relación que se da cara a cara entre el docente (tutor) y el aprendiz (tutorado) en la que se procura formar al tutorado para que pueda dimensionar las problemáticas que se le presentan en su vida escolar y llegue a resoluciones favorables que le aseguren el éxito escolar y profesional.

Considerando que la tutoría entreteje diversos elementos, actores, procesos, tiempos y espacios, resulta lógico advertir dificultades inherentes a su instrumentación. Estas, de acuerdo con su naturaleza, determinan los límites y los alcances de la tutoría, independientemente de que sea desarrollada por tutores nóveles o altamente experimentados. Ahora bien, vale la pena considerar la pregunta ¿qué debemos entender por dificultades de la tutoría? En un estudio realizado por Zazueta y Herrera (2009) con profesores de la Universidad Autónoma de Campeche sobre dificultades en la acción tutorial, determinaron que éstas se encuentran asociadas principalmente a la falta de definición de dicha actividad, problemas para atender ciertas demandas de los estudiantes (trastornos, adaptación, entre otros), así como un desconocimiento de las características precisas de intervención técnica (por ejemplo elaboración y/o aplicación de instrumentos de apoyo).

De acuerdo con lo señalado por Romo (2011), es necesario separarse de la idea de que la tutoría es un "remedio mágico" disponible en el momento, para abarcar y atender de manera absoluta cualquier problemática que presenten, ya sea los estudiantes, las instituciones, los docentes tutores como el proceso mismo. Por el contrario, la tutoría implica una intervención estructurada, planeada, que contempla una intervención oportuna a nivel cognitivo, social, cultural y existencial, centrada en un acompañamiento cercano, sistemático y permanente hacia el estudiante, de tal manera que se coadyuve a su integración y permanencia dentro de la institución.

Al traducir la tutoría desde una dimensión teórica hacia una dimensión práctica, es frecuente observar superposiciones de lo que se consideran acciones relativas propiamente de la tutoría y acciones concernientes a la práctica de la asesoría académica, o bien a la orientación psicopedagógica. En ese sentido, Fresán (2014) propone un deslinde de la tutoría con la asesoría y la orientación en educación superior. La asesoría está dirigida principalmente a la resolución de dudas teóricas o metodológicas a solicitud del alumno, sin que estas acciones se realicen dentro de una estructura formal, a diferencia de la tutoría que busca identificar problemáticas asociadas al desempeño del alumno para atenderlas de manera oportuna a través del seguimiento. 
En cuanto a orientación se refiere, se observan acciones que realiza generalmente un profesional de la psicología o psicopedagogía y que apoyan el trabajo de la tutoría al tratar casos específicos de intervención como lo serían las necesidades educativas especiales, elección vocacional, inclusión, desarrollo de habilidades del pensamiento, aspectos de personalidad, entre otros. Señala también que queda claro en este deslinde que las tareas de la tutoría apuntan hacia la toma de decisiones que tienen que ver con la vida y desarrollo académico del estudiante durante su formación profesional.

Sin embargo, para el Ministerio de Educación de la República del Perú (2007) la tutoría es considerada como una modalidad de la orientación educativa, le imprime la importancia de brindar un servicio integral de tipo socioafectivo, cognitivo y pedagógico que, a su vez, asegura la adecuada orientación a los estudiantes y con ello se previene la aparición de problemáticas. Los pilares que sustentan el trabajo conjunto de la tutoría y la orientación educativa son el currículo, el desarrollo humano y la relación tutorestudiante. Las áreas en las que interviene la tutoría son el área personal social, académica, vocacional, el área de salud corporal y mental, de ayuda social, de cultura y actualidad, y de convivencia y disciplina escolar. La tutoría se describe como formativa, preventiva, permanente, personalizada, integral, inclusiva, recuperada y no terapéutica.

Diversos autores señalan que el proceso formativo va acompañado tanto de ideas y razonamientos como de sentimientos y emociones por parte de los agentes (tutortutorado), por lo que la forma de ser que nos caracteriza influye en que seamos más o menos aptos para los diversos cometidos de la docencia universitaria, entre los que se encuentra la tutoría (Fullat y Henderson, citados en Serna, 2010 y Zabalza, 2007). Algunas cualidades que se vinculan a la función de tutoría y que se constituyen en facilitadores son, entre otras: disposición, apertura, flexibilidad, credibilidad (del componente ético profesional), calidez, empatía, cordialidad, todas éstas con una importante carga afectiva emocional.

Dada la relación tan cercana entre tutor y tutorado, y el apoyo integral que el estudiante recibe de su tutor, se hace necesaria la formación permanente de este último (Canales, 2004). Al respecto la ANUIES (citado en Serna, 2010) señala que el tutor debe mantenerse capacitado para realizar sus funciones, entre las que se encuentran: la revisión de la información académica y personal de los tutorados: trayectoria académica, estilos de aprendizaje, la identificación de actitudes o comportamientos que pudieran obstaculizar el desempeño del tutorado, el conocimiento del modelo educativo, la normatividad institucional aplicable al proceso formativo (servicio social, prácticas profesionales, titulación, movilidad e intercambio estudiantil, etc.). Es importante también que los tutores cuenten con habilidades para la planeación y la evaluación diagnóstica. 


\section{La tutoría y otras funciones de los docentes en la educación superior}

Si bien la función de tutoría ha sido hasta el momento la estrategia por excelencia a la que más impulso se le ha dado para atender las necesidades en materia de eficiencia terminal en la educación superior, existen otras acciones que se traducen en funciones que puede ejercer el docente universitario para lograr este objetivo. García-Cabrero et al. (2004) señalan que la docencia o práctica docente incluye a todas aquellas actividades relacionadas de forma directa con el proceso de enseñanza-aprendizaje de los alumnos. Los autores mencionan que dichas actividades son diversas y complejas, adoptando características particulares en función del área disciplinar, de las características inherentes al nivel educativo donde se insertan estas actividades y del contexto social y cultural de las poblaciones atendidas (García-Cabrero, Loredo y Carranza, 2008).

En el caso de las instituciones de educación superior se consideran otras funciones a desempeñar por parte del docente tales como la investigación, el servicio, la difusión, la tutoría, la asesoría y la supervisión. Aunque cada una de ellas persigue propósitos específicos y son actividades diferentes, pueden compartir competencias profesionales similares a las de la docencia.

Se incluye también como función docente a la orientación educativa y más recientemente se han incorporado el coaching educativo y la mentoría educativa.

A excepción de la supervisión, sin que quede exenta, todas las otras funciones docentes mencionadas han sido demandadas a la función docente de la tutoría. Es por ello que hacemos en este apartado una breve revisión de estas funciones, así como la presentación de una tabla que aporta a la síntesis.

\section{La función docente de supervisión}

Diversos autores en diferentes ámbitos profesionales han intentado definir la función docente de supervisión. Escartín, Lillo, Mira, Suárez y Palomar (2013), la definen como un proceso de acompañamiento en el desarrollo de las prácticas de intervención profesional con el fin de resolver dificultades, plantear interrogantes y reforzar las competencias que el alumnado debe adquirir para el ejercicio profesional.

Para Lobato (2007), la supervisión es un método de desarrollo profesional, un intento sistemático de mejorar la calidad de la práctica profesional. Mientras que el Council for Accreditation of Counseling and Related Educational Programs (CACREP) señala que la supervisión es una forma de instrucción, donde el docente lleva a cabo diferentes acciones, con el fin de regular y evaluar el rendimiento y la calidad del servicio prestado por una pasantía o práctica de los estudiantes (CACREP, 2009). Por su parte, García-Cabrero, Seda, García y Martínez (2013), señalan que la supervisión es un proceso permanente de acompañamiento gradual y continuado a los estudiantes, en el 
que, haciendo uso de diversas estrategias, se apoya a los alumnos en sus diferentes niveles de desempeño en relación con las competencias profesionales que están desarrollando.

\section{La función docente de asesoría académica}

La ANUIES (2002) señala que la asesoría académica es una actividad que llevan a cabo las IES, la cual cuenta con objetivos puntuales tales como: responder dudas en los contenidos académicos y reforzar los conocimientos, por lo que está centrada en los programas de estudio; responder a la petición del estudiante, lo que sucede la mayoría de las veces; tener una duración bastante temporal, se lleva a cabo en el área de trabajo del asesor y no requiere confidencialidad; y ocurrir o no dentro de la acción tutorial.

La asesoría académica es posible también observarla en la dirección de tesis o de proyectos de servicio social, así como en la coordinación de prácticas profesionales.

\section{La función docente de orientación educativa}

La orientación educativa es otra de las funciones docentes que intentan mejorar los aprendizajes esperados en el alumnado universitario. Al respecto Romo (2011), señala que la orientación educativa es un antecedente referencial de la tutoría que tiene nexos con experiencias de otros ámbitos profesionales. Boza, De la O y Salas (2001), señalan al orientador educativo como aquel profesional que contribuye a que la acción tutorial sea eficaz. Es importante recordar que la orientación educativa ha tenido dificultad para ser definida, ya que se le ha identificado con la orientación vocacional y también la asesoría que se ofrece a los alumnos para atender problemas de índole personal o social.

El concepto de orientación ha evolucionado a partir de aspectos como los objetivos que persigue, el momento y el lugar en que se lleva a cabo, así como el nivel formativo en que se ejerce. De acuerdo con Romo (2011), el concepto y operación de la orientación aparecen desde el siglo XX, referidos a la orientación vocacional. De esta forma en la evolución de la función docente de la orientación se observan tres etapas: la orientación escolar, la orientación profesional y la orientación psicopedagógica.

De acuerdo con Martínez (citado en Romo, 2011), actualmente se conocen cinco perspectivas de la orientación: la psicométrica, el modelo clínico, el modelo humanista, el modelo sociológico y el modelo psicopedagógico, también denominado constructivista. Esta última perspectiva de la orientación, además de estar centrada en el alumno, considera al centro escolar y a la comunidad como agentes condicionantes de procesos en el aprendizaje.

Es posible observar entonces que la función docente de orientar ha evolucionado del modelo clínico al modelo psicopedagógico, este último implica involucrar a toda la comunidad, desde los padres de familia, los profesores y por supuesto los estudiantes. De acuerdo con Sanz (citado en Romo, 2011), esta evolución de la función docente de 
la orientación dio lugar a la función docente de la tutoría. Es claro que los buenos profesores siempre ejercieron la función de orientar. Ahora es posible ver en la dinámica del proceso de tutoría que el docente en su función de tutor lleva a cabo generalmente acciones de orientación.

\section{El coaching educativo}

Martínez (2011), señala que el verbo to coach en español significa entrenar. Se considera que el aprendizaje se acelera cuando el entrenador es hábil. El coaching educativo se introduce en la educación superior a fines de la década de los noventa.

Se considera una estrategia pedagógica en la que el coach (tutor o docente) le facilita al estudiante la ejecución de acciones que pretenden potenciar las competencias y habilidades para que el coachee (la persona entrenada) desarrolle su propio potencial (Guido, 2011), a partir de la toma acertada de decisiones y la implementación de acciones concretas que se orientan al logro de los objetivos que, en el contexto universitario y personal, el coachee se haya planteado (Lárez, 2008).

Bou (citado en Lárez, 2008) define el coaching como un proceso "sistemático de aprendizaje, focalizado en la situación presente y orientado al cambio, en el que se facilitan unos recursos y unas herramientas de trabajo específicas, que permiten la mejora del desempeño en aquellas áreas que las personas demandan" (p. 11).

El coaching en la educación superior se puede aplicar en ámbitos como las evaluaciones, el apoyo a las reflexiones de los procesos de evaluación, autoevaluación y acreditación, en la gestión de investigaciones, para el análisis de problemas, en la identificación de grupos de interés, y en la evaluación de resultados de proyectos, entre otros.

De esta forma podemos puntualizar que el coaching en la educación superior se considera como una estrategia orientada al desarrollo y la consolidación de competencias profesionales en los participantes.

La siguiente tabla presenta a manera de síntesis las diversas funciones de la docencia expuestas en este apartado. 
Tabla 1. Definición, características, actores y procesos educativos de las diversas modalidades de la función docente

\begin{tabular}{|c|c|c|c|}
\hline Modalidad & Definición & Actores y procesos educativos & Palabras clave \\
\hline Tutoria & $\begin{array}{l}\text { Proceso de acompañamiento personal y } \\
\text { académico para mejorar el rendimiento, } \\
\text { solucionar problemas escolares, desarrollar } \\
\text { hábitos de estudio, trabajo, reflexión y } \\
\text { convivencia social (Fresán y Romo, 2011). }\end{array}$ & $\begin{array}{l}\text { Conjuga la presencia de un tutor (docente), } \\
\text { tutorado (alumno), plan curricular, trayectoria } \\
\text { académica. }\end{array}$ & $\begin{array}{l}\text { Acompañamiento } \\
\text { Trayectoria } \\
\text { Formación } \\
\text { Seguimiento }\end{array}$ \\
\hline $\begin{array}{l}\text { Asesoría } \\
\text { académica }\end{array}$ & $\begin{array}{l}\text { Herramienta educativa en la que el profesor } \\
\text { brinda atención, a manera de consulta y } \\
\text { fuera de su horario de clases, a los alumnos } \\
\text { con dudas respecto a un tema que domina } \\
\text { (Latapí citado en Fresán y Romo, 2011). }\end{array}$ & $\begin{array}{l}\text { Conjuga la presencia de un asesor, } \\
\text { asesorado, contenido temático y las } \\
\text { especificaciones en cuanto a la metodología } \\
\text { y compromisos de trabajo (Granja, 1989, } \\
\text { citado en Fresán y Romo, 2011). }\end{array}$ & $\begin{array}{l}\text { Consulta } \\
\text { Conocimiento } \\
\text { Dominio }\end{array}$ \\
\hline Orientación & $\begin{array}{l}\text { Descubrimiento y perfeccionamiento de } \\
\text { competencias personales y profesionales. Se } \\
\text { organiza a nivel individual o grupal y se } \\
\text { desarrolla mediante temáticas específicas. } \\
\text { Su duración es puntual, dirigida al } \\
\text { crecimiento de la persona para favorecer su } \\
\text { desarrollo profesional (Romo, 2011). }\end{array}$ & $\begin{array}{l}\text { Conjuga la presencia de un orientador que } \\
\text { apoya a los alumnos, tutores y profesores; } \\
\text { un tutor que orienta a un grupo/clase y un } \\
\text { tutorado que recibe orientaciones diversas } \\
\text { respecto a su proceso y formación } \\
\text { profesional. }\end{array}$ & $\begin{array}{l}\text { Actividad } \\
\text { procesual } \\
\text { Orientación en } \\
\text { grupo/clase }\end{array}$ \\
\hline Coaching & $\begin{array}{l}\text { Su propósito es acompañar al que desea } \\
\text { aprender, por un determinado tiempo, en el } \\
\text { logro de un objetivo definido y determinado } \\
\text { previamente, El alumno asume la total } \\
\text { responsabilidad y acepta al entrenador como } \\
\text { autoridad. Los esfuerzos se dirigen a la } \\
\text { tarea: retroalimentar y orientar las acciones } \\
\text { (Linares, 2014). }\end{array}$ & $\begin{array}{l}\text { Conjuga la presencia de un coach } \\
\text { (acompaña o entrena), un coachee (alumno), } \\
\text { un objetivo específico y un tiempo } \\
\text { determinado (Linares, 2014). }\end{array}$ & $\begin{array}{l}\text { Apoyo } \\
\text { Acompañamiento } \\
\text { Autoridad } \\
\text { Objetivo } \\
\text { determinado } \\
\text { Temporalidad }\end{array}$ \\
\hline Mentoría & $\begin{array}{l}\text { Proceso de retroalimentación continua de } \\
\text { ayuda y orientación entre pares para } \\
\text { potenciar el desempeño y rendimiento de un } \\
\text { alumno experimentado con otro u otros de } \\
\text { nuevo ingreso (Linares, 2014). }\end{array}$ & $\begin{array}{l}\text { Conjuga la presencia de un mentor (alumno } \\
\text { de un curso más avanzado), y un } \\
\text { mentorizado (alumnos de nuevo ingreso o } \\
\text { cursos iniciales), centrada en la atención de } \\
\text { necesidades y en promover su potencial. }\end{array}$ & $\begin{array}{l}\text { Retroalimentación } \\
\text { Tutoria entre pares } \\
\text { Orientación } \\
\text { Potencialización }\end{array}$ \\
\hline
\end{tabular}




\section{La mentoría como función docente}

Con el ánimo de clarificarlas, en el apartado anterior se han referido y contrastado funciones docentes diversas como la enseñanza en el aula; la docencia/supervisión en escenarios de formación profesional; la asesoría, la orientación, el coaching y la tutoría. En este listado es notoria la ausencia de una de las funciones docentes escasamente retomada, nos referimos a la mentoría, que rara vez ha sido abordada, aun cuando se le ha considerado clave para la formación de los estudiantes.

Desde la antigüedad se ha atribuido a las personas con mayor experiencia la responsabilidad de guiar e instruir a los menos experimentados. El origen de la idea de mentoría se remonta a la Grecia Antigua, aparece por primera vez plasmada en La Odisea atribuida a Homero. En uno de sus pasajes se describe cómo Ulises, rey de Ítaca, antes de su partida a la guerra de Troya, deposita en su amigo Mentor la guía y educación de Telémaco, su único hijo.

Siglos después, Fenelón François (1690) autor del libro Las aventuras de Telémaco describe a Mentor como el consejero de Telémaco y es en esta obra que aparece por primera vez el término "mentor" con un significado cercano al que se le atribuye en los tiempos modernos. La obra, utilizada con fines pedagógicos (para el aprendizaje del francés), fue muy exitosa por más de dos siglos durante los cuales se tradujo a diferentes idiomas y se le atribuye la posibilidad del origen del término como se le conoce actualmente.

Conceptualización y funciones de la mentoría

La conceptualización y las prácticas de mentoría han cambiado a lo largo del tiempo, en que han surgido diferentes enfoques y modelos explicativos. A continuación, se muestra la recopilación realizada por Valverde, Ruiz de Miguel y Romero (2004) de las definiciones de mentoría de diferentes autores: 
Tabla 2. Definiciones de mentoría

\begin{tabular}{|c|c|c|c|}
\hline Autor & Año & Definición & Énfasis \\
\hline Allen & 1998 & $\begin{array}{l}\text { Es la ayuda que una persona proporciona a otra para } \\
\text { que progrese en su conocimiento, su trabajo o su } \\
\text { pensamiento. }\end{array}$ & Ayuda para el progreso general. \\
\hline $\begin{array}{l}\text { Council of } \\
\text { Graduate } \\
\text { Schools of } \\
\text { Michigan } \\
\text { University } \\
\end{array}$ & 1999 & $\begin{array}{l}\text { Es una relación formal e intencionada, entre una } \\
\text { persona con mucha experiencia y habilidades en un } \\
\text { área o ámbito determinado, y una persona novata en } \\
\text { dicha área o ámbito, a través del cual se desarrollan } \\
\text { procesos de orientación y guía. }\end{array}$ & $\begin{array}{l}\text { Formal e intencionada. } \\
\text { Proceso de ayuda y guía. }\end{array}$ \\
\hline Faure & 1999 & $\begin{array}{l}\text { Es una relación a largo plazo que cubre las necesidades } \\
\text { de desarrollo, ayuda a conseguir el máximo potencial y } \\
\text { beneficia a todas las partes (mentor, mentorizado y } \\
\text { organización). }\end{array}$ & $\begin{array}{l}\text { Responde a necesidades. } \\
\text { Beneficia a todas las partes. } \\
\text { Largo plazo. }\end{array}$ \\
\hline $\begin{array}{l}\text { Starcevich } \\
\text { y Friend }\end{array}$ & 1999 & $\begin{array}{l}\text { La mentorización consiste en un proceso de } \\
\text { construcción y beneficio mutuo (entre los miembros } \\
\text { implicados en dicho proceso), para ayudar a desarrollar } \\
\text { los conocimientos y conductas del nuevo alumnado, } \\
\text { profesorado, o trabajador, por parte de un alumno de } \\
\text { curso avanzado, o de un profesor/ trabajador más } \\
\text { experimentado. }\end{array}$ & $\begin{array}{l}\text { Proceso constructivo. } \\
\text { Desarrollo de conocimientos y } \\
\text { conductas. }\end{array}$ \\
\hline Fletche & 2000 & $\begin{array}{l}\text { Es una relación de uno a uno, que proporciona guía y } \\
\text { apoyo a un tutelado al que le facilita una época de } \\
\text { transición en su vida. No es sinónimo de clonar, porque } \\
\text { significa desarrollar virtudes individuales para maximizar } \\
\text { su potencial personal y profesional. }\end{array}$ & $\begin{array}{l}\text { Ayuda en periodos de transición. } \\
\text { No es clonar. } \\
\text { Uno a uno. }\end{array}$ \\
\hline Parsloe & 1999 & $\begin{array}{l}\text { Su propósito es apoyar y alentar a la gente en la mejora } \\
\text { de su propio aprendizaje para maximizar su potencial, } \\
\text { desarrollar sus habilidades, y mejorar sus actuaciones } \\
\text { para convertirse en la persona que quieren llegar a ser. }\end{array}$ & $\begin{array}{l}\text { Mejora del aprendizaje. } \\
\text { Desarrollo del potencial. }\end{array}$ \\
\hline Soler & 2003 & $\begin{array}{l}\text { La estrategia del mentoring es un proceso por el cual } \\
\text { una persona con más experiencia (el mentor) enseña, } \\
\text { aconseja, guía y ayuda a otra (el tutelado) en su } \\
\text { desarrollo personal y profesional, invirtiendo tiempo, } \\
\text { energía y conocimientos. }\end{array}$ & $\begin{array}{l}\text { Desarrollo en general. } \\
\text { Guía y ayuda. }\end{array}$ \\
\hline
\end{tabular}

Fuente: Tomado de Valverde, Ruiz de Miguel y Romero (2004)

Para Carr (1999) "Un mentor es simplemente alguien que te ayudó a aprender algo que no hubieras aprendido, o que hubieras aprendido más lentamente o con mayor dificultad, de haber tenido que hacerlo enteramente por tu cuenta" (1999, p. 6).

Este autor refiere a dos modelos o enfoques: el de mentoría natural y el de mentoría intencional (Carr, 1999). En el modelo de mentoría natural, también conocido como informal o no intencional, el mentor generalmente es una persona mayor, con más experiencia y ajeno al ámbito familiar del "asociado" o mentorizado. El vínculo que se establece entre ambas personas obedece a factores diferentes, entre ellos, que se identifican personal o profesionalmente o que hay admiración, esto sostiene el vínculo y 
generalmente puede ser a largo plazo hasta que una de las partes decide concluirlo. Este tipo de mentoría no establece propósitos, temporalidad ni planeación alguna. Por su naturaleza tampoco implica la asignación de mentor, ni valoración o seguimiento.

En el caso del enfoque o modelo de mentoría intencional, descrito también como formal o sistemático, se selecciona, prepara y asigna un mentor, se establecen objetivos y proyectos o actividades específicas para abordar y lograr resultados identificables en diferentes planos del desarrollo del mentorizado (Carr, 1999; MENTOR/National Mentoring Partnership, 2005). Este tipo de mentoría también se lleva a cabo exitosamente entre pares de estudiantes 0 de colegas con diferentes niveles de experticia o pericia, en una estructura de interacción flexible y simétrica (Douglass, Smith L., y Smith J., 2013). Se realiza en modalidad individual o grupal y se determina claramente la duración (horas/semanas/meses) y temporalidad (por ejemplo, un cuatrimestre, un semestre). Tanto en la mentoría individual como en la mentoría de pares, el conocimiento en el tema y las habilidades para comunicarse de manera efectiva son los que más aprecian mentores y mentorizados (Douglass et al., 2013).

Diferentes autores coinciden al definir la mentoría formal como la relación de apoyo entre alguna persona con mayor experiencia (mentor) y alguien con menor experiencia (mentorizado). Señalan que se constituye en mentoría formal porque los mentores son asignados y se comprometen a seguir los propósitos y estructura de un programa determinado (Ragins y Cotton, citados en Larose, Cyrenne, Garceau et al., 2011).

Kochan (2002, citado en Kochan 2013) por su parte, define la mentoría como:

Dos o más individuos formando voluntariamente una relación mutua de respeto y confianza, centrada en objetivos que abarcan las necesidades y que promueven el potencial del mentorizado, tomando en cuenta las necesidades del mentor y el contexto que sea funcional para ambos.

Se han descrito diversas funciones de un mentor, éstas abarcan temas como conocimiento, habilidades de comunicación y soporte emocional. Larose et al. (2011) comentan que en un primer estadio, el mentor debe lograr que su mentorizado realice la transición del nivel escolar anterior a la Universidad, se refieren por tanto a una mentoría inicial. Posteriormente, a lo largo de la carrera, el mentor debe fomentar la autonomía, ayudar a sus mentorizados en la planeación y priorización de sus metas, a estudiar para sus exámenes, para reunirse con sus profesores, enseñarles técnicas de estudio, discutir problemas acerca de su carrera y fomentar la visita a lugares de trabajo de su profesión que les ayuden a entender qué quieren ser y cómo pueden lograrlo (Larose et al., 2011).

\section{Relación entre tutoría y mentoría}

En este punto es importante poder reflexionar sobre la comparación entre funciones del ejercicio de la tutoría y el de la mentoría, más que deslindar estos conceptos entre sí, se trata de observar su complementariedad, la tutoría (junto con la asesoría académica) 
descansa en una figura institucional, el docente; por su parte, la mentoría posibilita la inclusión de un nuevo elemento encargado de realizar un proceso de acompañamiento, el alumno experto.

En ambos casos la relación es intencionada y se establece en un marco de interacción sistemático (planeado y con objetivos), sin embargo, un punto diferencial es que, para el caso de la mentoría, el beneficio formativo es mutuo, impacta a ambos actores (mentor y mentorizado), los dos aprenden: uno en la medida en que enseña o facilita y el otro en tanto que es guiado en la apropiación de los contenidos. Podemos atribuir este resultado a la paridad del uso de códigos de comunicación correspondientes a su grupo etario.

Bajo esta perspectiva, la relación entre el proceso de tutoría y de mentoría es bidireccional, por un lado, la tutoría encuentra en la mentoría una fuente de apoyo y su relación se sustenta en la canalización y el seguimiento, siendo el espacio de la tutoría donde se pueden identificar necesidades específicas de acompañamiento particularizado en el estudiante y establecer estrategias de atención, haciendo uso del recurso de la mentoría, lo cual supone que mentor y mentorizado pueden establecer una agenda de trabajo a la luz de la observación y del respaldo de un tutor, dejando lugar claro está, a la independencia del proceso de mentoría y a la evolución de éste.

En segundo término, la mentoría representa un espacio desde el cual el mentor fomenta la importancia y los beneficios que representa el utilizar todos los sistemas de acompañamiento que la institución ofrece (tutoría, asesoría académica), particularmente a la población estudiantil de reciente ingreso. "Los programas de mentoría entre iguales pretenden favorecer la adaptación universitaria del alumnado de nuevo acceso, facilitándole acogida, integración, información y asesoramiento para su integración académica y social (...) para desarrollar conjuntamente competencias generales y específicas dentro de un contexto de aprendizaje colaborativo y de un clima de confianza" (Velasco y Benito, citado en Fernández-Salinero, 2014).

Tal y como lo señalan Casado, Lezcano y Colomer (2015), se debe apostar por una conciliación entre los planes de acción tutorial y otras modalidades de acompañamiento, aludiendo a que el programa de mentoría debe estar integrado en los planes de acción tutorial en las universidades.

Algunas experiencias de programas de mentoría en universidades europeas y estadounidenses

La Universidad Complutense de Madrid cuenta con un Programa de Mentorías (peer mentoring) cuyo objetivo se centra en facilitar la integración de estudiantes de nuevo ingreso y estudiantes extranjeros, no solo a la vida académica, sino a la dinámica social que prevalece en la institución, a través de potenciar las habilidades sociales, de relación, orientación y liderazgo. Aunado a esto, los mentores favorecen la comprensión de todos 
los procedimientos administrativos inherentes a la formación del nuevo estudiante universitario.

En el portal web del programa de la Complutense se despliegan una caracterización y los beneficios para las dos figuras participantes, un plan de formación para los mentores y una guía para la adscripción de los estudiantes que lo soliciten. Asimismo, se integra un sistema de seguimiento a cargo de los coordinadores del programa y un amplio mecanismo de evaluación que incluye una valoración integral de los resultados por parte de los mentores, los alumnos mentorizados o telémacos (término utilizado en el programa para referirse a los estudiantes de nuevo ingreso) y profesores tutores, a través de instrumentos colgados en la red (Universidad Complutense de Madrid, s. f.).

Por su parte, la Universidad Politécnica de Madrid (UPM) en un primer momento contó con el Proyecto Mentor de la Escuela Técnica Superior de Ingeniería de Telecomunicación (ETSI), cuyo objetivo era coadyuvar en la supervivencia del alumno de nuevo ingreso a través del contacto y relación con estudiantes de segundo ciclo que fungían como mentores. La estructura de este sistema de tutorías funcionaba de manera vertical y horizontal y operaba de manera integrada y dinámica bajo la tutela de un profesor tutor y el desarrollo de actividades entre mentores y alumnos.

En un ejercicio de valoración del proyecto de Mentoría de la ETSI se observó una apreciación favorable del programa por parte de los estudiantes beneficiados, quienes señalaron haberse sentido orientados social, administrativa y académicamente. A raíz de ello se implantó la Red Temática de Mentoría Universitaria en once centros de dicha institución, con el objetivo de considerar a la mentoría como una "estrategia de innovación educativa eficaz y eficiente" para promover una adaptación exitosa y expedita del alumno al nuevo entorno formativo; y, por otra parte, para buscar la generación de conocimientos sólidos que sustenten los beneficios de la aplicación sistemática de programas de mentoría (Sánchez, Almendra, Jiménez, Melcón y Macías, 2009).

En 1990 se crea en Estados Unidos la Asociación MENTOR/National Mentoring Partnership, que por más de dos décadas ha desarrollado programas y recursos para realizar acciones de mentoría en diferentes ámbitos y poblaciones con alcance en América Latina.

Esta asociación define a la mentoría como "una relación estructurada y de confianza que les permite a las personas jóvenes reunirse con individuos interesados que ofrecen orientación, apoyo y ánimo con el fin de desarrollar las competencias y el carácter del aprendiz" (MENTOR/National Mentoring Partnership, 2005, p. 9).

Esta Asociación plantea 5 tipos de mentoría:

1) Mentoría tradicional: en este tipo de mentoría la relación es de uno a uno, en la que el adulto se constituye en el mentor de una persona joven para apoyar en sus intereses y necesidades de desarrollo. 
2) Mentoría grupal: de un adulto con un grupo pequeño de hasta cuatro jóvenes, a partir de intereses y metas comunes de los mentorizados.

3) Mentoría en equipo: varios mentores con experiencia y conocimientos en diferentes campos brindan colaborativamente la mentoría a grupos pequeños de jóvenes para incidir en diferentes aspectos de su formación; se sugiere que la proporción sea de un adulto por cada 4 jóvenes como máximo.

4) Mentoría de compañeros: se trata de mentoría entre pares, en la que jóvenes con mayor conocimiento o habilidades interesados en adoptar este rol, proporcionan mentoría a sus compañeros o a otros jóvenes.

5) Mentoría electrónica: la mentoría en línea, a través de correos electrónicos o plataformas educativas, es una alternativa complementaria para agilizar la comunicación, o bien cuando se tienen ubicaciones geográficas diferentes.

Los diferentes programas de mentoría coinciden en los resultados positivos que pretenden alcanzar con los mentorizados, pero se diferencian en dos tipos según las metas específicas que establecen: a) programas con metas focalizadas al desarrollo personal-social de los jóvenes, b) programas con metas orientadas al logro académico (Douglass, Smith L., y Smith J., 2013).

Las actividades se establecen en el plan de mentoría a partir de las necesidades de apoyo manifestadas por el mentorizado, quien protagoniza y codirige el proceso con su mentor. Es decir, el mentorizado tiene un papel activo en la determinación y desarrollo de las actividades específicas de apoyo que requiere, manifestando sus necesidades, intereses y expectativas de la mentoría. De esta manera, el actor principal del proceso de mentoría en la interacción mentor-mentorizado es el estudiante (Valverde, Ruiz de Miguel y Romero, 2004; Douglass, Smith L., y Smith J., 2013).

Dawson (2014) identificó en la literatura 16 elementos básicos para la planeación y conducción de la mentoría, a partir de una exhaustiva revisión y análisis de diferentes fuentes, que pueden resumirse e identificarse en dos modelos: el de Instrucción suplementaria (SI, por sus siglas en inglés), que es un programa consolidado de aprendizaje entre pares, y el Esquema de Enseñanza Asistida por Pares (PATS), que es un programa reciente de mentoría con profesores universitarios como mentorizados.

Todos los elementos referidos por Dawson (2014) han sido retomados por los autores de este trabajo para delinear una propuesta de programa de mentoría docente suplementaria (PMDS) dirigida a estudiantes universitarios, con la intención de que a partir de la experiencia que se obtenga de su implementación se avance en la creación de un modelo integral de mentoría, en el cual se trabaja actualmente. Se hace énfasis en la necesidad de crear la figura de Mentor en el contexto institucional universitario, la cual se propone pueda ser asignada a docentes o a estudiantes para que desarrollen la función de mentoría entre pares; ya sea mentoría a pares docentes que se inician en la enseñanza, o mentoría a pares estudiantes menos aventajados. 
Se pretende que esta figura de mentor colabore llevando a cabo actividades específicas complementarias a la acción de los profesores que realizan funciones docentes como la tutoría o asesoría, por mencionar algunas, y que requieren que sus tutorados o asesorados reciban mentoría en aspectos específicos para avanzar en su formación y prevenir, minimizar o evitar la reprobación, el bajo rendimiento, la deserción o la postergación de la terminación de estudios y titulación.

Para que esta figura de mentor logre sus propósitos, debe contar además con programas de actualización y formación permanente en las áreas de conocimiento en las que orientará la mentoría suplementaria, además del reconocimiento y el soporte institucional necesario para desarrollar las siguientes funciones:

1) Diseñar y planificar el programa de mentoría con el alcance y temporalidad acordes a los ciclos escolares e institucionales, a partir también de las necesidades detectadas, población a mentorar y recursos con que se cuente. Desde la planificación plantear los objetivos e intenciones de la mentoría, establecer las líneas de acción a emprender, así como los mecanismos de evaluación y seguimiento de la mentoría que se prevé desarrollar y realizar los ajustes pertinentes a la planeación con base en la práctica directa de la mentoría.

2) Prever e implementar durante el proceso de la mentoría acciones concretas para el desarrollo y/o fortalecimiento de las competencias disciplinares específicas del mentorizado, mediante el trabajo con los contenidos académicos específicos que se le dificulten, con problemáticas de bajo rendimiento o reprobación de asignaturas identificadas en su trayectoria escolar o manifestadas por el estudiante.

3) Contribuir, aun cuando el foco de su actuación durante la mentoría estará centrado en los contenidos específicos a trabajar, al desarrollo de actitudes y valores concretos y claramente establecidos que le aporten al mentorizado mayor capacidad para la convivencia armónica y el bienestar en su vida personal, social, académica y profesional. Las acciones y funciones del mentor contemplan, por lo tanto, participar en el desarrollo de competencias personales y competencias para la comunicación interpersonal, por ejemplo para la reflexión de sus propias acciones, para la toma de decisiones y la solución de problemas. Esto de manera complementaria a la actuación de otros profesionales o mentores involucrados.

4) Manejar los incidentes y la resolución de problemas durante el proceso de mentoría. Analizar las causas y plantear conjuntamente con el mentorizado las formas de afrontar y resolver la situación que genere incomodidad o conflicto que pudiera desestabilizar la relación mentor-mentorizado, propiciando la posible ruptura del vínculo. Actúa tomando las medidas necesarias en co-responsabilidad con el mentorizado. En caso necesario, también toma medidas para dar por concluida la relación de mentoría y canalizarlo. 
5) Implementar la estrategia de evaluación anticipada durante la planificación de la mentoría. Implica la valoración al inicio, durante y al cierre del proceso de mentoría, del impacto que la mentoría tiene o tuvo en el mentorizado, con los procedimientos que determine. El mentor además se responsabilizará de la aplicación de las herramientas de evaluación que institucionalmente se proporcionen para evaluar y dar seguimiento al logro de los objetivos propuestos.

6) Comunicar resultados de la mentoría a los docentes involucrados directa 0 indirectamente, durante y al cierre del proceso de mentoría.

A manera de consideración general se enuncian diez pasos clave para el apoyo entre universitarios de acuerdo con lo que establecen Casado, Lezcano y Colomer (2015), quienes señalan lo siguiente:

- El primer paso para establecer un sistema de acompañamiento de mentoría es preparar de manera adecuada el programa, considerando espacios tiempos, recurso humano y personal.

- El segundo paso se refiere a la búsqueda de alianzas que aseguren la participación activa de los estudiantes.

- El tercer paso implica la identificación de mecanismos de capacitación y selección de mentores, dando lugar al cuarto paso: generar un sistema de incentivos para el personal que funja como mentor.

- El quinto paso hace referencia a la implementación de la formación de los mentores, especificando temas, tiempos y expertos.

- El sexto paso subraya la intervención de los profesores tutores y su habilitación para dar seguimiento al programa.

- El séptimo paso incluye acciones de capacitación para los estudiantes de nuevo ingreso en relación a la operatividad del programa.

- El octavo paso atiende al proceso operativo-administrativo para la asignación de alumnado mentorizado a su nuevo mentor o mentora.

- El noveno hace alusión a la conciliación de la mentoría con los planes de acción tutorial y otras modalidades de acompañamiento y orientación.

- El décimo paso consiste en establecer mecanismos claros para el seguimiento y la evaluación del programa que permitan identificar necesidades de mejora.

\section{CONSIDERACIONES FINALES}

El señalamiento de las diferentes funciones asumidas desde el ejercicio docente como formas paralelas a la enseñanza que permiten guiar la formación integral de los estudiantes supone la integración de diversas competencias, herramientas y espacios desde los cuales se brinda un acompañamiento de calidad, que contribuye al fortalecimiento de los perfiles profesionales dentro de un programa de estudios. 
El repaso en este desarrollo teórico sobre las diversas tareas docentes permite diferenciarlas entre sí y demuestran la evolución en cuanto a su noción y operación. Es así que, desde el surgimiento de la actividad orientadora, se ha pasado por el ejercicio de la tutoría, la supervisión, la asesoría y el coaching educativo, para aterrizar en la práctica de la mentoría como propuesta fundamental de este trabajo.

Si bien el acompañamiento estudiantil (visualizado formalmente en los modelos educativos) considera dentro de sus objetivos elementales el fomentar la autonomía de pensamiento, la toma de decisiones de impacto para la vida, así como el desarrollo académico y profesional de los individuos implica también un mayor anclaje a factores de carácter personal que inciden en los resultados formativos. Por tanto, se hace preciso ampliar su marco de impacto, incorporando nuevos elementos, actores y acciones que signifiquen una innovación en el campo de la formación, como lo sería el extender el ejercicio de la tutoría a la tarea de la mentoría en las instituciones de educación superior.

A diferencia de la supervisión (que valora el rendimiento), de la asesoría académica (que refuerza conocimientos), de la orientación educativa (que atiende al desarrollo psicopedagógico) y al coaching educativo (que se apoya en el entrenamiento de competencias profesionales), la mentoría además de integrar estos atributos amplía el alcance de estas guías considerando la identificación personal y profesional entre el actor experimentado y el actor novato, brindando la oportunidad de que el considerado experto pueda ser el propio docente o bien un par estudiantil, lo que a juicio de los autores le significa un valor agregado a la propuesta de incluir el proceso de mentoría dentro de los programas formativos.

Una posible línea endeble sería pasar de la mentoría natural a la mentoría formal, lo que representa un reto para la puesta en práctica de un programa como el de la Mentoría Docente Suplementaria (PMDS) desde la cual los docentes y estudiantes (aventajados) fungirían como mentores bajo un programa concebido con propósitos y mecanismos de seguimiento y evaluación establecidos y una proporción equilibrada entre mentor y cantidad de estudiantes asignados, lo que implica un desafío para algunas instituciones ya que la relación entre tutor y cantidad de tutorados es sumamente desproporcionada, representando una dificultad de alcance en la mayoría de los casos.

Como se señaló, la mentoría tiene tareas equiparables con la tutoría, lo que la distingue es la idea del apoyo entre pares basado en el interés genuino por el desarrollo y logro de un fin educativo por parte del aprendiz, que como en todas las funciones señaladas con anterioridad es el actor central del proceso.

De ahí pues que la esencia de esta aportación sea la de orientar la propuesta de la inclusión de la mentoría como actividad complementaria y paralela a la tutoría, que sin duda conlleva varias áreas de oportunidad, no solo en la capacitación y habilitación permanente de mentores sino en la conceptualización de un modelo como tal, con procesos, tiempos, espacios y mecanismos de evaluación definidos y particularmente la de dar entrada a una nueva figura guía: el propio estudiante. 


\section{REFERENCIAS}

Asociación Nacional de Instituciones de Educación Superior. (2000). La educación superior en el Siglo XXI. Líneas estratégicas de desarrollo. México D. F., México: Asociación Nacional de Instituciones de Educación Superior.

Asociación Nacional de Instituciones de Educación Superior. (2002). Programas Institucionales de Tutoría. Una propuesta de la ANUIES para su organización y funcionamiento en las instituciones de educación superior. México D. F., México: Serie Investigaciones.

Boza, Á., De la O, M., y Salas, M. (2001). Orientación y tutoría: conceptualización. En Boza, Á. (Ed.), Ser profesor, ser tutor. Orientación educativa para docentes (pp. 19-56). Huelva, España: Hergué.

Canales, E. (2010). El perfil del tutor académico. Recuperado de https://www.uaeh.edu.mx/investigacion/productos/4614/

Canales, E. (2004). La formación de tutores académicos en educación superior. En T. C. Barrón (Coord.). Currículum y actores: Diversas miradas (pp.47-59). México D.F, México: Universidad Nacional Autónoma de México.

Carr, R. (1999). Alcanzando el futuro: El papel de la mentoría en el nuevo milenio. Victoria, Canadá: Peers Resources. Recuperado de http://www.mentors.ca/spanish1.pdf

Casado, R., Lezcano, F., Colomer, J. (2015). Diez pasos clave en el desarrollo de un programa de mentoría universitaria para estudiantes de nuevo ingreso. Revista Electrónica Educare, 19(2), 168-172.

Council for Accreditation of Counseling and Related Educational Programs. (2009). CACREP Accreditation Manual. Alexandria, VA: Author.

Dawson, P. (2014). Beyond a definition: Toward a framework for designing and specifying mentoring models. Educational Researcher, 43(3), 137-145.

Douglass, A., Smith L., D., y Smith J. L. (2013). An Exploration of characteristics of effective undergraduate peer-mentoring relationships. Mentoring y Tutoring: Partnership in Learning, 21(2), 219-234.

Escartín, M., Lillo, M., Mira, J., Suárez, E., y Palomar, M. (2013). El proceso de supervisión en las prácticas de trabajo social. En M. Tortosa, J. Álvarez y N. Pellín (Coords.), XI Jornadas de Redes de Investigación en Docencia Universitaria. Retos de futuro en la enseñanza superior: docencia e investigación para alcanzar la excelencia académica (pp.679-691). Alicante, España: Universidad de Alicante. Recuperado de http://rua.ua.es/dspace/handle/10045/31305

Fernández-Salinero, C. (2014). La tutoría universitaria en el escenario del espacio europeo de educación superior: perfiles actuales. Teoría Educativa, 26, 1-20.

Fresán, M., y Romo, A. (2011). Programas institucionales de tutoría. Una propuesta de la ANUIES. México D.F., México: Asociación Nacional de Instituciones de Educación Superior.

Fresán, M. (2014). Una mirada autocritica: los programas institucionales de tutorías. En A. Romo (Coord.), Los programas institucionales de tutoría: actores, procesos y contextos (pp. 27-48). México D.F., México: Asociación Nacional de Instituciones de Educación Superior. 
García-Cabrero, B., Loredo, J., Luna, E., Pérez, C., Reyes, R., Rigo, M. A. y Rueda, M. (2004). Algunas consideraciones sobre los aspectos teóricos involucrados en la evaluación de la docencia. En M. Rueda y F. Díaz-Barriga, La evaluación de la docencia en la universidad. Perspectivas desde la investigación y la intervención profesional (pp.13-61).México D.F., México: Plaza y Valdés.

García-Cabrero, B., Loredo, J. y Carranza, G. (2008). Análisis de la práctica educativa de los docentes: pensamiento, interacción y reflexión. Revista electrónica de investigación educativa, Número especial. Recuperado de http://redie.uabc.mx/index.php/redie/article/view/200/345

García-Cabrero, B., Seda, I. García, H. y Martínez, S. (septiembre de 2013). La Evaluación de Competencias en Educación Superior. Ponencia presentada en el VIII Congreso Internacional de Evaluación Formativa en Docencia Universitaria: Universidad de Valladolid, Segovia, España.

Guido, E. (2011). Coaching en la educación superior. Semanario de la Universidad de Costa Rica. Recuperado de https://semanariouniversidad.com/opinion/coaching-en-la-educacin-superior/

Islas, D., y González, J. (2014). Tutoría para la calidad de vida y las relaciones humanas. México, D.F., México: Umbral.

Kochan, F. (2013). Analyzing the Relationships Between Culture and Mentoring. Mentoring y Tutoring: Partnership in Learning, 21(4), 412-430.

Lara, B. (Coord.). (2009). La tutoría académica en educación superior: modelos, programas y aportes. Guadalajara, México: Universidad de Guadalajara.

Lárez, J. (2008). El coaching educativo como estrategia para potenciar el éxito durante la etapa de desarrollo y culminación del trabajo especial de grado. Sapiens, 9(2), 219-234. Recuperado de http://www.redalyc.org/pdf/410/41011837013.pdf

Larose, S., Cyrenne, D., Garceau, O., Harvey, M., Guay, F., Godin, F., Deschenes, C. (2011). Academic mentoring and dropout prevention for students in math, science and technology. Mentoring and Tutoring: Partnership in Learning, 19(4), 419-439. https://doi.org/10.1080/13611267.2011.622078

Linares, G. (2014). El Coaching en la educación superior. Revista Iberoamericana de producción académica y gestión educativa, 1, 1-10.

Lobato, C. (2007). La supervisión de la práctica profesional socioeducativa. Revista psicodidáctica, 12(1), 29-50.

Martínez, J. (2011). Coaching y liderazgo. Contribuciones a la economía. Recuperado de http://www.eumed.net/ce/2011a/jamg3.htm

Martínez, Y., Montaño, A. y Ortiz, M. (2011). Intervención psicopedagógica en estudiantes de la licenciatura en ciencias de la comunicación y licenciatura en psicología. En A. Montaño, Y. Martínez, M. Ortiz y S. Ponce (Coords.), La psicopedagogía como referente educativo en la UABC (pp.19-53). Mexicali, México: Universidad Autónoma de Baja California

MENTOR/National Mentoring Partnership. (2005). Cómo construir un programa de mentoría exitoso. Utilizando los Elementos de la práctica efectiva. Alexandria, VA: The National Mentoring Partnership. Recuperado de: http://www.mentoring.org/downloads/mentoring_414.pdf 
Ministerio de Educación de la República del Peru. (2007). Manual de tutorias y orientacion educativa. Ministerio de Educación. Programa educación básica para todos. Peru. Recuperado de http://www.minedu.gob.pe/minedu/archivos/a/002/02-bibliografia-comun-a-ebr-eba-y-etp/3manual-de-tutoria-y-orientacion-educativa.pdf

Romo, A. (2004). La incorporación de los programas de tutoría en las instituciones de educación superior. Mexico D.F., México: Asociación Nacional de Instituciones de Educación Superior.

Romo, A. (Coord.) (2011). La tutoría. Una estrategia innovadora en el marco de los programas de atención a estudiantes. Mexico D.F., México: Asociación Nacional de Instituciones de Educación Superior.

Romo, A. (2014). Los programas institucionales de tutoría: actores, procesos y contextos. Mexico D.F., México: Asociación Nacional de Instituciones de Educación Superior.

Sánchez, A., Almendra, A., Jiménez, F., Melcón, M., y Macías, J. (2009). Proyecto Mentor en la Universidad Politécnica de Madrid: un sistema de mentoría para la acogida y orientación de los alumnos de nuevo ingreso. Sistemas, Cibernética e Informática. 6(1), 64-71.

Serna, A. (2010). La tutoría académica desde la mirada del alumno. Mexicali, México: Universidad Autónoma de Baja California.

UNESCO. (1998). Declaración Mundial sobre la Educación Superior en el Siglo XXI: Visión y acción. Conferencia Mundial sobre la Educación Superior. Recuperado de: http://www.unesco.org/ducation/educprog/wche/declaration_spa.htm

Universidad Complutense de Madrid. (s.f.). Programa de Mentorías. Recuperado de http://www.ucm.es/mentorias

Valverde, A., Ruiz de Miguel, C., y Romero, S. (2004). Innovación en la orientación universitaria: la mentoría como respuesta. Contextos educativos: Revista de educación, 6-7, 87-112.

Zabalza, M. A. (2007). Competencias docentes del profesorado universitario. Calidad y desarrollo profesional. Madrid, España: Narcea.

Zazueta, Ma. E. y Herrera, L. (2009). Dificultades en la acción tutorial reportadas por los tutores de la Facultad de Odontología de la Universidad Autónoma de Campeche. Recuperado de https://www.uv.mx/encuentrotutorias/MEMORIAS\%20DEL\%20IV\%20ENCUENTRO.pdf 